\title{
Treatment of Comorbid Obesity and Major Depressive Disorder: A Prospective Pilot Study for their Combined Treatment
}

\author{
Lucy F. Faulconbridge, ${ }^{1}$ Thomas A. Wadden, ${ }^{1}$ Robert I. Berkowitz, ${ }^{1,2}$ \\ Melissa E. Pulcini, ${ }^{1}$ and Thomas Treadwell ${ }^{3}$ \\ ${ }^{1}$ Center for Weight and Eating Disorders, Department of Psychiatry, University of Pennsylvania, School of Medicine, \\ Philadelphia, PA 19104, USA \\ ${ }^{2}$ Department of Child and Adolescent Psychiatry and Behavioral Sciences, Children's Hospital of Philadelphia, Philadelphia, \\ PA 19104, USA \\ ${ }^{3}$ Center for Cognitive Therapy, Department of Psychiatry, University of Pennsylvania, School of Medicine, Philadelphia, \\ PA 19104, USA
}

Correspondence should be addressed to Lucy F. Faulconbridge, lucyhf@mail.med.upenn.edu

Received 20 October 2010; Revised 4 March 2011; Accepted 21 March 2011

Academic Editor: Terry Huang

Copyright (c) 2011 Lucy F. Faulconbridge et al. This is an open access article distributed under the Creative Commons Attribution License, which permits unrestricted use, distribution, and reproduction in any medium, provided the original work is properly cited.

\begin{abstract}
Background. Obese individuals who suffer from major depressive disorder are routinely screened out of weight loss trials. Treatments targeting obesity and depression concurrently have not been tested. Purpose. To test the short-term efficacy of a treatment that combined behavioral weight management and cognitive behavioral therapy (CBT) for obese adults with depression. Methods. Twelve obese females diagnosed with major depressive disorder received weekly group behavioral weight management, combined with CBT for depression, for 16 weeks. Weight, symptoms of depression, and cardiovascular disease (CVD) risk factors were measured at baseline and week 16. Results. Participants lost 11.4\% of initial weight and achieved significant improvements in symptoms of depression and CVD risk factors. Conclusions. Obese individuals suffering from major depressive disorder can lose weight and achieve improvements in symptoms of depression and CVD risk factors with 16 weeks of combined treatment. A larger randomized controlled trial is needed to establish the efficacy of this treatment.
\end{abstract}

\section{Introduction}

Depression and obesity are both associated with an increased risk of cardiovascular disease [1-3] and with marked impairments in quality of life $[4,5]$. These two disorders frequently cooccur, and each presents a risk for the development of the other $[6,7]$. Despite their cooccurrence, depression and obesity rarely are treated concurrently, and there are no models for providing such combined therapy. The absence of care can be traced to concerns raised by two studies, conducted in the 1950s, which concluded that dieting precipitated (or exacerbated) symptoms of depression $[8,9]$. Such concerns, however, have little current empirical support. Studies of obese, mildly depressed individuals treated in behavioral weight loss trials have revealed satisfactory weight loss and improvements in symptoms of depression, as measured by self-report instruments [10]. Studies are lacking of obese individuals with major depression who are provided behavioral treatment for both their obesity and their depression, and in whom mood is assessed by diagnostic interview.

The present pilot study addressed this important treatment gap by providing obese individuals with major depressive disorder (MDD) a 16-week group treatment program that combined two evidence-based approaches-cognitive behavioral therapy (CBT) for depression and behavioral treatment for obesity. Our primary hypotheses were that, at the end of treatment, participants would achieve (1) a mean weight loss $\geq 5 \%$ of initial weight and (2) a clinically significant reduction in depression, defined by a $40 \%$ decrease on the Hamilton Depression Rating Scale (HDRS) [11]. Our secondary hypotheses were that participants would 
achieve significant reductions in cardiovascular (CVD) risk factors including waist circumference, blood pressure, and lipids.

\section{Methods}

Enrollment was open to men and women, ages $18-72 \mathrm{yr}$, who had a body mass index (BMI) of $30-45 \mathrm{~kg} / \mathrm{m}^{2}$ and suffered from MDD. They also were required to have at least one additional risk factor for CVD, which included elevations in waist circumference, blood pressure, fasting blood glucose levels, triglycerides, or reduced HDL cholesterol, as defined by the National Cholesterol Education Program (Adult Treatment Panel III) [12]. In addition, type 2 diabetes or medications for any of these conditions were considered CVD risk factors. Exclusion criteria included a weight loss of $\geq 5 \%$ of initial weight in the prior 6 months, current individual or group psychotherapy, or occurrence of a cardiovascular event in the last 12 months. Participants taking selective serotonin reuptake inhibitors had to have been on a stable dose for at least 3 months prior to enrollment; individuals taking other types of antidepressants were excluded (due to their potential effects on weight). A total of 253 applicants responded to advertisements in the local media, of whom 202 were successfully contacted for a phone screening interview. (The other 51 individuals could not be reached.) Of these 202, 166 were excluded following the phone screen, the majority of whom did not meet criteria for a current depressive episode. The remaining 36 applicants were invited to attend an in-clinic screening visit at which the details of the study were explained, written informed consent was obtained, and a clinical psychologist met with participants to assess the presence of MDD using a Structured Clinical Interview for the DSM-IV (SCID) [13]. Of these 36, 12 did not attend the visit, 5 decided they were not interested after learning more about the study, 4 did not meet the depression criteria, and 1 required treatment for another psychiatric disorder. Fourteen participants attended a second screening visit, which included collection of a fasting blood sample. One participant later determined that she would not be able to attend the group meeting when scheduled. A second participant withdrew from the program because of a family emergency, immediately before treatment began. This left 12 participants who began the trial. The study's protocol and procedures were approved by the University of Pennsylvania's Institutional Review Board.

Participants attended 16 weekly, 90-min group sessions (in groups of 6 participants) led by a clinical psychologist (LF). Half of each session was devoted to behavioral weight management skills, based on Brownell's LEARN Program for Weight Management [14]. Participants were weighed each week, given calorie goals (i.e., $1200-1500 \mathrm{kcal} / \mathrm{d}$ ) based on their initial weight, encouraged to increase exercise gradually to $180 \mathrm{~min} / \mathrm{wk}$, and taught skills such as self-monitoring and stimulus control. The other half of each session consisted of group CBT for depression, based on Bieling et al.'s group treatment for depression [15]. Participants were introduced to the cognitive model of depression and taught to identify and modify their negative automatic thoughts and core beliefs. Participants were weighed at each group session and completed the Beck Depression Inventory-II (BDI-II) [16] to track the course of symptoms of depression. The BDIII is a 21-item questionnaire that assesses mood over the previous 2 weeks. Total scores range from 0 to 63 , with higher values indicating greater symptoms of depression. Scores of 0-13 reflect minimal (i.e., subclinical) symptoms, whereas values of $14-19,20-29$, and $\geq 30$ indicate mild, moderate, and severe symptoms of depression, respectively.

Participants completed outcomes assessments at baseline and week 16 at which weight, height, and other characteristics were measured. Body weight was measured on a digital scale (Detecto, model 6800A) while participants were dressed in light clothing, without shoes. Height was measured using a wall-mounted stadiometer (Veeder-Root, Elizabethtown, NC). Waist circumference and blood pressure were assessed using methods described previously [17]. Fasting blood samples were obtained (and analyzed by Quest Diagnostics, Horsham, PA). Symptoms of depression were assessed using the HDRS by a trained, independent clinician who was masked to the aims of the study. The HDRS was the study's primary outcome for assessing changes in mood.

Changes in weight, HDRS scores, and CVD risk factors (from baseline to week 16) were assessed by withinsubjects repeated-measures $t$-tests. Eleven of 12 participants completed the study. One participant dropped out at week 12 because of her mother's declining health; her data were carried forward from week 11 (i.e., her last in-clinic weight and BDI-II score). A second participant became pregnant at week 14 . She continued attending group until the end of treatment, but no data after her pregnancy are included in the analyses. A third participant showed significant worsening in symptoms of depression at week 13 , at which point treatment was intensified and therefore no longer consistent with the study protocol. She received individual treatment sessions with the study psychologist (LF), was evaluated by the study psychiatrist (RB), and was then referred to appropriate adjunctive treatment. (The participant continued to attend groups until the end of the study, but her data following treatment intensification, at week 13, were not included in data analysis.).

\section{Results}

At baseline, the 12 participants had a mean $( \pm$ SD) age of $45.5 \pm 9.3 \mathrm{yr}$, weight of $94.5 \pm 15.9 \mathrm{~kg}$ and BMI of $34.9 \pm$ $4.7 \mathrm{~kg} / \mathrm{m}^{2}$. Six participants were non-Hispanic white, five were African American, and one was of multiple races. The baseline HDRS score was $20.2 \pm 3.3$, indicating moderate to severe symptoms of depression.

As shown in Figure 1(a), the nine participants who completed the full study lost $10.4 \pm 4.9 \mathrm{~kg}$ over 16 weeks $(P<.001)$, equal to a reduction of $11.4 \pm 5.9 \%$ of initial weight $(P<.001)$. (All of the nine participants lost at least $5 \%$ of initial weight.) The mean HDRS score decreased from $20.4 \pm 3.7$ to $9.3 \pm 4.9$, equal to a mean reduction of $11.1 \pm 4.8$ points $(P<.001)$. Figure $1(\mathrm{~b})$ presents changes in HDRS 


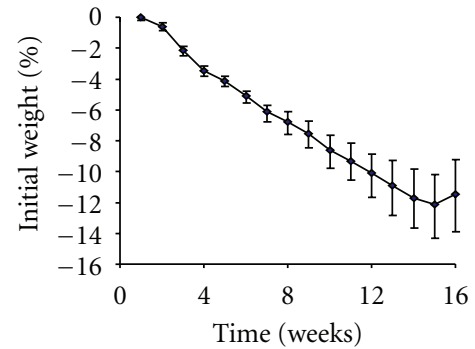

(a)

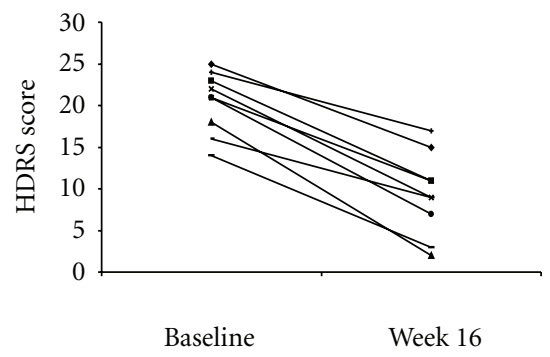

(b)

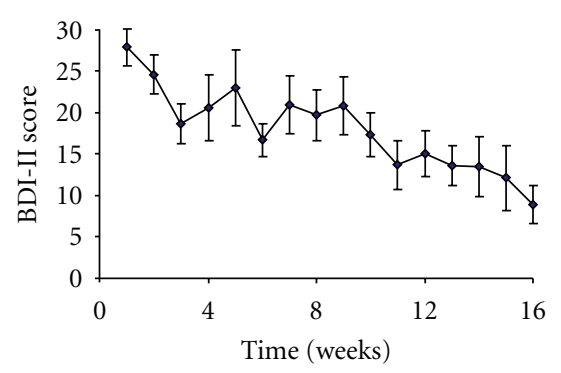

(c)

FIGURE 1: (a) shows mean change in percent of initial weight over 16 weeks of treatment $(n=9)$. (b) presents changes in HDRS scores for each participant from baseline to week $16(n=9)$. (c) shows mean change in BDI-II score over 16 weeks of treatment $(n=9)$.

TABLE 1: Mean ( \pm SD) changes in cardiometabolic risk factors $(N=$ 9).

\begin{tabular}{|c|c|c|c|c|}
\hline & Baseline & Week 16 & Change & $P$-value \\
\hline Waist $(\mathrm{cm})$ & $104.2 \pm 10.5$ & $93.6 \pm 12.7$ & $10.6 \pm 6.4$ & .001 \\
\hline Systolic BP & $118.4 \pm 14.4$ & $110.4 \pm 12.4$ & $8.1 \pm 8.8$ & .025 \\
\hline Diastolic BP & $72.0 \pm 8.2$ & $63.3 \pm 8.7$ & $8.7 \pm 5.3$ & .001 \\
\hline \multicolumn{5}{|l|}{ Cholesterol } \\
\hline Total (mg/dL) & $213.2 \pm 27.4$ & $182.7 \pm 22.2$ & $30.6 \pm 29.3$ & .014 \\
\hline HDL (mg/dL) & $68.9 \pm 13.8$ & $61.0 \pm 12.7$ & $7.9 \pm 10.5$ & .055 \\
\hline $\mathrm{LDL}(\mathrm{mg} / \mathrm{dL})$ & $127.0 \pm 24.8$ & $105.4 \pm 19.7$ & $21.6 \pm 21.3$ & .001 \\
\hline $\begin{array}{l}\text { Triglycerides } \\
\text { (mg/dL) }\end{array}$ & $86.7 \pm 34.2$ & $81.1 \pm 35.8$ & $5.6 \pm 27.6$ & .562 \\
\hline Glucose (mg/dL) & $87.9 \pm 5.0$ & $82.8 \pm 5.0$ & $5.1 \pm 6.4$ & .042 \\
\hline Insulin $(\mathrm{uIU} / \mathrm{mL})$ & $7.1 \pm 5.2$ & $5.1 \pm 4.6$ & $2.0 \pm 3.4$ & .118 \\
\hline Hs-CRP (mg/L) & $5.3 \pm 5.2$ & $4.9 \pm 3.5$ & $0.4 \pm 3.7$ & .748 \\
\hline
\end{tabular}

BP: blood pressure; HDL: high density lipoprotein; LDL: low-density lipoprotein; Hs-CRP: high sensitivity C-reactive protein. $P$ values based on repeated-measures $t$-tests.

scores for each participant. Using the criterion of an HDRS score $\leq 8$ [18], six (of nine) participants $(67 \%)$ were classified as responders (i.e., full remission of depression). All nine participants who completed the study achieved at least a $40 \%$ reduction in HDRS scores, and five achieved at least a 50\% reduction. As shown in Figure 1(c), BDI-II scores declined from $27.9 \pm 7.3$ to $8.9 \pm 7.6$ at week 16 , a decline of $19.0 \pm 5.3$ points $(P<.001)$.

Data were examined at the time of last observation for the three participants who did not complete the study. They lost a mean of $7.3 \pm 1.5 \mathrm{~kg}$, equal to a reduction of $7.5 \pm 3.5 \%$ of initial weight $(P<.001)$. The mean HDRS score decreased from $19.3 \pm 2.1$ to $12.0 \pm 14.0$. Two of the three participants achieved an $88.2 \%$ and $100 \%$ reduction in HDRS scores, respectively; the third participant showed an increase of $4 \%$. The three participants reported a mean reduction on the BDI-II of $7.6 \pm 16.9$ points (i.e., an improvement in symptoms of depression).

As shown in Table 1, significant improvements were observed in the CVD risk factors examined, with the exception of triglycerides and HDL cholesterol (the latter declined rather than increased, as is typical with shortterm caloric restriction [19]). Framingham Risk scores [12] declined from $4.2 \%$ to $1.7 \% 10$-year risk $(P<.01)$.

Two participants were taking selective serotonin reuptake inhibitors throughout the study. No differences in changes in symptoms of depression were observed between these two participants and the rest of the group. They did, however, lose a smaller percentage of initial weight (6.5\% versus $11.3 \%)$, but the limited sample size prevents statistical analysis.

\section{Discussion}

This is the first study of which we are aware to combine behavioral weight management for obesity with CBT for depression for obese individuals diagnosed with major depressive disorder. Participants who completed the study lost $11.4 \pm 5.9 \%$ of initial weight in 16 weeks-a loss comparable to that achieved in nondepressed individuals after 16 weeks of behavioral treatment [20]. This finding contradicts concerns that depressed individuals will not be able to lose as much weight as their non-depressed counterparts $[21,22]$.

As a group, participants also achieved significant improvements in symptoms of depression, as shown by the reduction on the HDRS scores. Baseline scores indicative of moderate to severe symptoms of depression fell at week 16 to levels indicative of mild symptoms, and two thirds of the participants who completed the study achieved full remission of depression. Only one participant experienced worsening mood symptoms. This individual had a longstanding history of severe, treatment-resistant depression (including an inpatient hospitalization in the last decade). Of note, she lost $9.2 \%$ of her initial weight in spite of her worsening affect, and she chose to complete the study. Thus, for the great majority of our obese individuals with MDD, moderate weight loss combined with CBT was associated with improvements, rather than worsening, in symptoms of depression.

Concerns that depressed individuals cannot adhere to treatment recommendations or will drop out of treatment [23] were not supported by this study. Eleven out of 12 participants continued to come to group sessions until the end of the study (with usable data from nine participants), 
and participants attended $89.6 \%$ of possible treatment sessions.

Prior studies have established that moderate weight losses ( $\sim 5 \%$ of initial weight) are sufficient to improve CVD risk factors $[24,25]$. Our participants achieved significant improvements in waist circumference, diastolic and systolic blood pressure, and LDL, and total cholesterol. HDL cholesterol declined significantly (rather than increased) at week 16 , as usually observed with short-term weight loss [19]. HDL cholesterol typically increases over the long term, after caloric restriction is terminated.

This study had numerous limitations, including a small sample size and a lack of appropriate control and comparison conditions. Our combined therapy needs to be evaluated against behavioral weight loss alone, as well as CBT for depression alone, to determine whether the combination treatment is superior to each monotherapy. A follow-up assessment of 6-12 months also is needed to provide data about the durability of changes in both weight and symptoms of depression. A 1-year randomized controlled trial, with a larger sample size, is the critical next step in establishing the efficacy of this treatment. In addition, our promising results require replication in a representative sample. The current participants may reflect a subset of highly motivated treatment-seeking individuals. These limitations notwithstanding our combined treatment offers hope for the large numbers of depressed, obese individuals who remain without appropriate care.

\section{Acknowledgment}

Preparation of this paper was supported by DK065018.

\section{References}

[1] A. K. Ferketich, J. A. Schwartzbaum, D. J. Frid, and M. L. Moeschberger, "Depression as an antecedent to heart disease among women and men in the NHANES I study. National Health and Nutrition Examination Survey," Archives of Internal Medicine, vol. 160, no. 9, pp. 1261-1268, 2000.

[2] S. M. Grundy, B. Hansen, S. C. Smith, J. I. Cleeman, and R. A. Kahn, "Clinical management of metabolic syndrome: report of the American Heart Association/National Heart, Lung, and Blood Institute/American Diabetes Association conference on scientific issues related to management," Circulation, vol. 109, no. 4, pp. 551-556, 2004.

[3] N. T. Nguyen, X. M. Nguyen, J. B. Wooldridge, J. A. Slone, and J. S. Lane, "Association of obesity with risk of coronary heart disease: findings from the National Health and Nutrition Examination Survey, 1999-2006," Surgery for Obesity and Related Diseases, vol. 6, no. 5, pp. 465-469, 2010.

[4] K. R. Fontaine and I. Barofsky, "Obesity and health-related quality of life," Obesity Reviews, vol. 2, no. 3, pp. 173-182, 2001.

[5] R. L. Spitzer, K. Kroenke, M. Linzer et al., "Health-related quality of life in primary care patients with mental disorders. Results from the PRIME-MD 1000 study," Journal of the American Medical Association, vol. 274, no. 19, pp. 1511-1517, 1995.

[6] G. Hasler, D. S. Pine, A. Gamma et al., "The associations between psychopathology and being overweight: a 20-year prospective study," Psychological Medicine, vol. 34, no. 6, pp. 1047-1057, 2004.

[7] R. E. Roberts, G. A. Kaplan, S. J. Shema, and W. J. Strawbridge, "Are the obese at greater risk for depression?" American Journal of Epidemiology, vol. 152, no. 2, pp. 163-170, 2000.

[8] A. Keys, J. Brozek, A. Henschel, F. Michelson, and H. L. Taylor, The Biology of Human Starvation, University of Minnesota Press, Minneapolis, Minn, USA, 1950.

[9] A. J. Stunkard, “The dieting depression; incidence and clinical characteristics of untoward responses to weight reduction regimens," The American Journal of Medicine, vol. 23, no. 1, pp. 77-86, 1957.

[10] L. F. Faulconbridge, T. A. Wadden, R. I. Berkowitz et al., "Changes in symptoms of depression with weight loss: results of a randomized trial," Obesity, vol. 17, no. 5, pp. 1009-1016, 2009.

[11] M. Hamilton, "A rating scale for depression," Journal of Neurology, Neurosurgery, and Psychiatry, vol. 23, pp. 56-62, 1960.

[12] "Third report of the National Cholesterol Education Program (NCEP). Expert panel on detection, evaluation, and treatment of high blood cholesterol in adults (adult treatment panel III) final report " Circulation, vol. 106, no. 25, pp. 3143-3421, 2002.

[13] R. L. Spitzer, J. B. Williams, M. Gibbon, and M. B. First, "The structured clinical interview for DSM-III-R (SCID). I: history, rationale, and description," Archives of General Psychiatry, vol. 49, no. 8, pp. 624-629, 1992.

[14] K. D. Brownell, The Learn Program for Weight Management, American Health Publishing, 2000.

[15] P. M. Bieling, R. E. McCabe, and M. M. Antony, CognitiveBehavioral Therapy in Groups, The Guilford Press, 2006.

[16] A. T. Beck, R. A. Steer, R. Ball, and W. Ranieri, "Comparison of Beck depression inventories -IA and -II in psychiatric outpatients," Journal of Personality Assessment, vol. 67, no. 3, pp. 588-597, 1996.

[17] The Practical Guide: Identification, Evaluation and Treatment of Overweight and Obesity in Adults, NHLBI Obesity Education Initiative, 2000.

[18] E. Frank, R. F. Prien, R. B. Jarrett et al., "Conceptualization and rationale for consensus definitions of terms in major depressive disorder. Remission, recovery, relapse, and recurrence," Archives of General Psychiatry, vol. 48, no. 9, pp. 851-855, 1991.

[19] R. E. Andersen, T. A. Wadden, S. J. Bartlett, B. Zemel, T. J. Verde, and S. C. Franckowiak, "Effects of lifestyle activity vs structured aerobic exercise in obese women: a randomized trial," Journal of the American Medical Association, vol. 281, no. 4, pp. 335-340, 1999.

[20] T. A. Wadden, M. L. Butryn, and C. Wilson, "Lifestyle modification for the management of obesity," Gastroenterology, vol. 132, no. 6, pp. 2226-2238, 2007.

[21] S. Pagoto, J. S. Bodenlos, L. Kantor, M. Gitkind, C. Curtin, and Y. Ma, "Association of major depression and binge eating disorder with weight loss in a clinical setting," Obesity, vol. 15, no. 11, pp. 2557-2559, 2007.

[22] E. Ludman, G. E. Simon, L. Ichikawa et al., "Does depression reduce the effectiveness of behavioral weight loss treatment?" Behavioral Medicine, vol. 35, no. 4, pp. 126-134, 2010.

[23] M. M. Clark, R. Niaura, T. K. King, and V. Pera, "Depression, smoking, activity level, and health status: pretreatment predictors of attrition in obesity treatment," Addictive Behaviors, vol. 21, no. 4, pp. 509-513, 1996. 
[24] M. Espeland, X. Pi-Sunyer, G. Blackburn et al., "Reduction in weight and cardiovascular disease risk factors in individuals with type 2 diabetes: one-year results of the look AHEAD trial," Diabetes Care, vol. 30, no. 6, pp. 1374-1383, 2007.

[25] W. C. Knowler, E. Barrett-Connor, S. E. Fowler et al., "Reduction in the incidence of type 2 diabetes with lifestyle intervention or metformin," The New England Journal of Medicine, vol. 346, no. 6, pp. 393-403, 2002. 


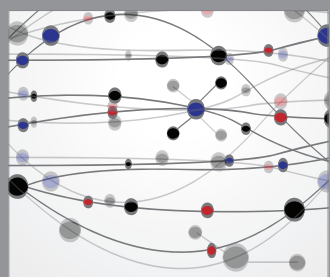

The Scientific World Journal
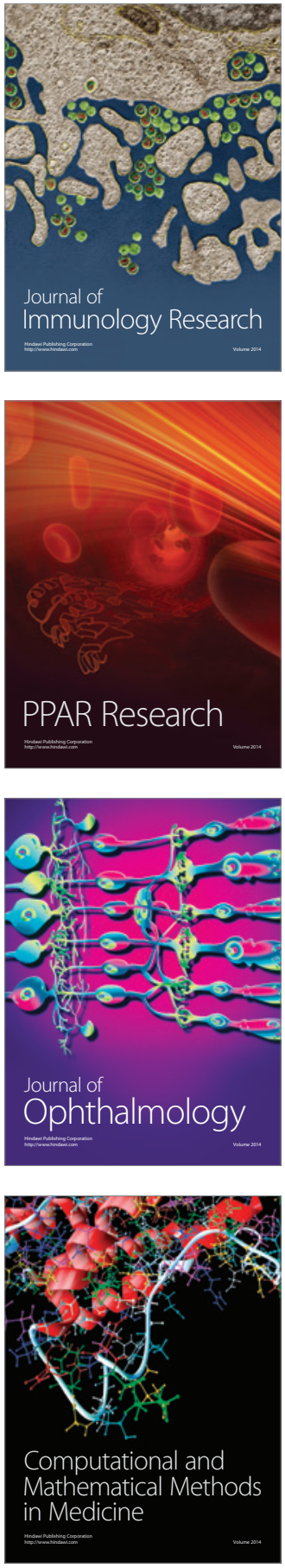

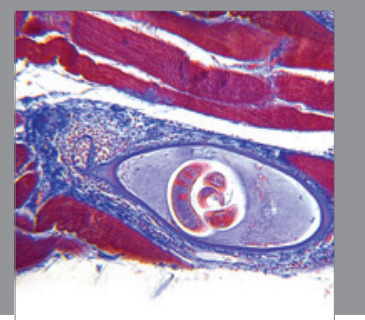

Gastroenterology

Research and Practice
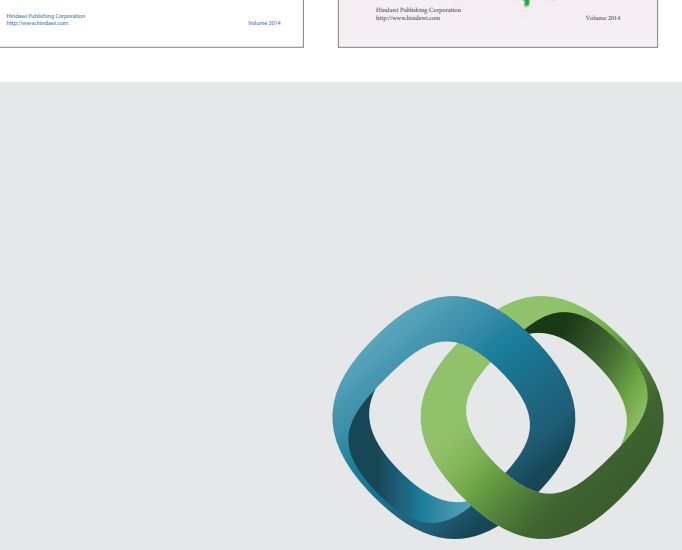

\section{Hindawi}

Submit your manuscripts at

http://www.hindawi.com
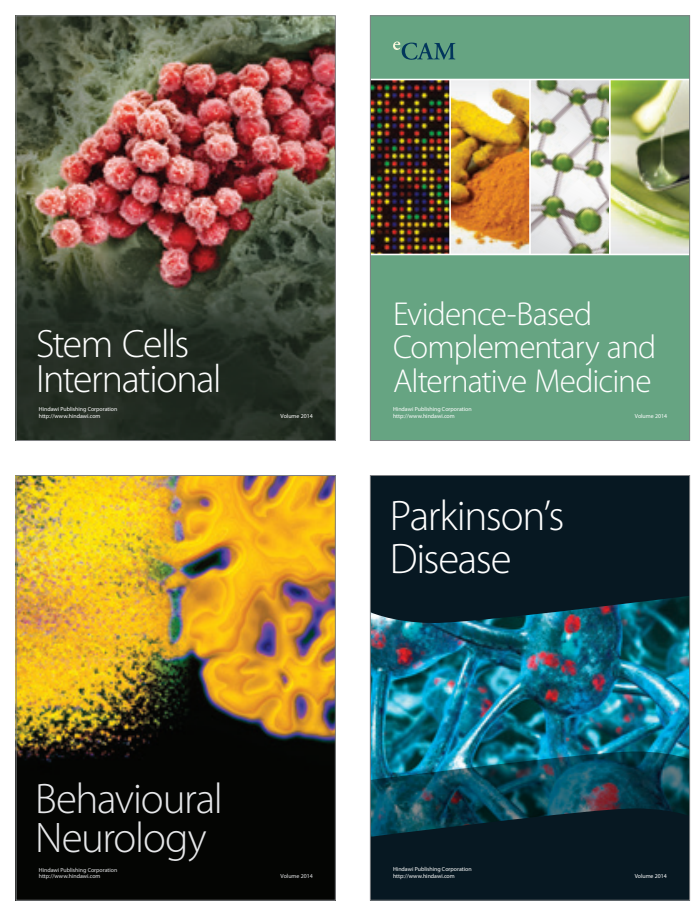

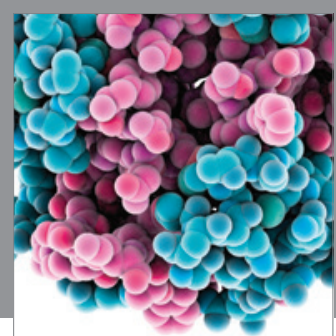

Journal of
Diabetes Research

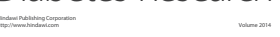

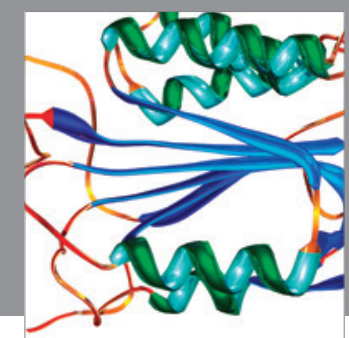

Disease Markers
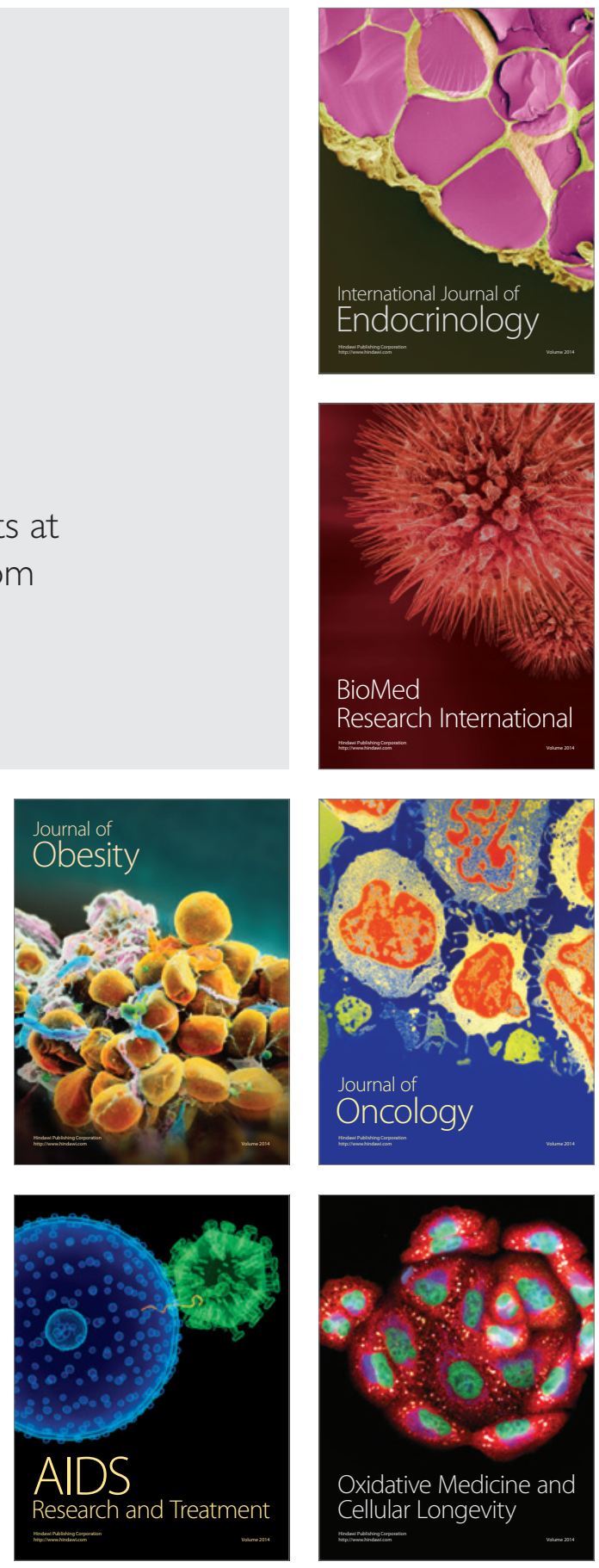